\title{
1 On the whereabouts of HIV-1 cellular entry and its fusion ports
}

2 G. Maria Jakobsdottir ${ }^{1}$, Maro Iliopoulou ${ }^{1}$, Rory Nolan ${ }^{1}$, Luis Alvarez ${ }^{1}$, Alex A. Compton ${ }^{2}$ and

3 Sergi Padilla-Parra ${ }^{1,3, *}$

4 1-Wellcome Trust Centre for Human Genetics, Cellular Imaging, University of Oxford,

5 Oxford, $U K$

6 2-HIV-1 Dynamics and Replication Program, National Cancer Institute, 1050 Boyles Street.

$7 \quad$ Frederick, MD 21702-1201, USA

8 3-Division of Structural Biology, University of Oxford, The Henry Wellcome Building for

9 Genomic Medicine, Headington, Oxford OX3 7BN, UK

10

11

12

13

14

15

16

17

*Corresponding author: Sergi Padilla-Parra, spadilla@well.ox.ac.uk

\begin{abstract}
HIV-1 disseminates to diverse tissues through different cell types and establishes long-lived reservoirs. The exact cellular compartment where fusion occurs differs depending on the cell type and mode of viral transmission. This implies that HIV-1 may modulate a number of common host cell factors in different cell types. In this review, we evaluate recent advances on the host cell factors playing an important role in HIV-1 entry and fusion. New insights from restriction factors that inhibit virus-cell fusion in vitro may contribute the development of future therapeutic interventions. Collectively, novel findings underline the need for potent, host-directed therapies that disrupt the earliest stages of the virus life cycle and preclude the emergence of resistant viral variants.
\end{abstract}


Tens of millions of people are infected with the human immunodeficiency virus type-1 (HIV1) worldwide, and a similar number have died from the spectrum of acquired immune deficiency syndrome (AIDS) related diseases (Gallo, 2016). Despite the ability to administer anti-retroviral therapy (ART) to prevent HIV-1 morbidity and mortality in AIDS patients, this therapeutic approach does not eradicate the virus and remains inaccessible in many countries burdened with the majority of global infections (Medlock et al., 2017). Basic research focusing on the interaction of HIV-1 with numerous cell types is important to understand the mechanisms of virus persistence, transmission, and pathogenesis. Existing ART treatment lowers viremia to near undetectable levels but fails to eliminate HIV-1 from infected individuals, presumably due to the presence of latent viral reservoirs in $\mathrm{CD}^{+} \mathrm{T}$ cells, and other cell types (Boxes 1 and 2)(Gandhi et al., 2010). Indeed, other cell types are susceptible to HIV-1 infection, such as macrophages and other hematopoietic cells of the myeloid lineage (Simmons et al., 1997), and can also contribute to the establishment of chronic infection. While a well-established non-human primate model using Simian Immunodeficiency Virus (SIV) has revealed the importance of myeloid cell infection (Honeycutt et al., 2017; Laguette et al., 2012), a better understanding of how human tissue macrophages respond to HIV-1 infection is required to more effectively engineer strategies for virus eradication. The role of dendritic cells (DCs) in HIV-1 infection is clearly of interest, as they facilitate virus transmission to T cells despite being refractory to HIV-1 fusion and productive infection themselves (Geijtenbeek et al., 2000b). It is possible that HIV-1 circumvents direct infection of DCs to prevent activation of an innate immune response. In this review, we consider recent advances in the study of HIV-1 entry in different cellular contexts. Indeed, the application of new imaging techniques (like super-resolution (Chojnacki et al., 2012), spectral imaging (Jones et al., 2017; Russell et al., 2017; Zaitseva et al., 2017) or Cryo-EM (Lee et al., 2016; Wu et al., 2010)) have leaded to recent discoveries that highlight the importance of the point of HIV-1 fusion or how HIV-1 hijacks host proteins for entry (also termed host restriction factors). Here, we describe important advances on the molecular mechanisms leading to HIV-1 fusion in different cellular contexts:, macrophages, dendritic cells (DCs) and T cells and the role of different host restriction factors during entry. Interestingly, the point of fusion (i.e. plasma membrane versus endocytic compartments) differs depending on the cell line. 
Endocytosis has surfaced as a potentially important route of HIV-1 entry (de la Vega et al., 2011; Miyauchi et al., 2009a). Several studies have dealt with this hypothesis in different biological contexts. In particular, evidence in macrophages provides a strong case for viruscell fusion occurring within endosomal compartments (Li et al., 2017; Marechal et al., 2001; Russell et al., 2017). Macrophages exhibit high endocytic activity relative to T cells (Mellman, 1996) and HIV-1 uptake following endocytosis could be a route to productive infection in this cell lines (Russell et al., 2017). In an initial study documenting the observation that HIV-1 could enter macrophages via endocytosis Carter and co-workers (Carter et al., 2011); employed a pharmacological approach utilizing inhibitors for caveolin-1-related endocytosis, micropinocytosis, actin rearrangements and Rho GTPases, finding that most likely HIV-1 entry into macrophages proceeds via an endocytic pathway related to micropinocytosis. Furthermore, genetic approaches have been used to demonstrate that the overexpression of CD4 at the surface of induced pluripotent stem cell (iPSC)-derived human macrophages (PSCmacrophages) can increase HIV-1 fusion but not HIV-1 infection efficiency, as evidenced from viral DNA expression assays (van Wilgenburg et al., 2014). Indeed, HIV-1 was demonstrated to fuse within endosomes in PSC-macrophages via a Dynamin-2 (DNM2), Rasrelated $\mathrm{C} 3$ botulinum toxin substrate 1 (RAC1 GTPase) and Pak-dependent (kinase) pathway. The authors, measured fusion and reverse transcription in PSC-macrophages expressing LCK tyrosine protein kinase, -which tethered CD4 in the cell surface altering its normal endocytic rate increasing CD4 expression 3-fold. This led to a significant increase in HIV-1 fusion and reverse transcription but strikingly, productive HIV-1 infection was unaffected, implying that productive infection by HIV-1 in macrophages happens via an endocytic uptake pathway.

Recent evidence utilizing real-time single virus tracking and imaging of double labeled HIV-1 virions demonstrates that HIV-1 can enter primary derived macrophages and fuse from endosomal compartments within these cells (Russell et al., 2017) (Li et al., 2017). Moreover, the data seem to indicate that HIV-1 can enter the cell via clathrin-mediated endocytosis as HIV-1 fusion was inhibited when using dynasore (Kirchhausen et al., 2008), a small molecule DNM2 inhibitor, and is guided toward Rab5a-positive early endosomal compartments where fusion can then occur as visualized by advanced optical microscopy (Russell et al., 2017) (Li et al., 2017). One plausible hypothesis for this would be that, in macrophages, the fusion reaction might be initiated by HIV-1 Env upon engagement of CD4 and CCR5, but yet, cannot be completed in the plasma membrane because the increased endocytic rate relative to $\mathrm{T}$ cells. 
Therefore, the transition towards full fusion might occur once HIV-1 virions are harboured within early endosomes as previously shown in TZM-bl cells in (de la Vega et al., 2011) and primary macrophages in (Li et al., 2017; Russell et al., 2017). However, the finding that HIV1 fusion could be arrested in macrophages using dynasore, a small molecule DNM2 inhibitor, does not necessarily imply that HIV-1 necessarily enters the cell through clathrin-mediated endocytosis because the target of the drug, GTPase dynamin-2 (DNM2), might also be implicated in other aspects of virus fusion (Jones et al., 2017; Wen et al., 2016).

In brief, recent studies show evidence for endosomal HIV-1 fusion in primary derived macrophages and PSC-macrophages. The occurrence of HIV-1 fusion in the plasma membrane of macrophages, however, cannot be totally discarded. The potential endocytic route for productive HIV-1 entry and fusion is not fully elucidated yet; as there is some discrepancy in the literature about this (micropinocytosis (Marechal et al., 2001; van Wilgenburg et al., 2014) versus clathrin-mediated endocytosis (Li et al., 2017)).

\section{Interactions between HIV-1 and dendritic cells}

Because of their physiological role as professional antigen presenting cells, and their localization at mucosal surfaces, it has been proposed that DCs are among the first cells to encounter HIV-1 during transmission (Piguet and Steinman, 2007). DCs express relatively low levels of the CD4 receptor and CCR5/CXCR4 co-receptors, which is thought to be one reason why they are not regularly infected by HIV-1 (Parrish et al., 2012). Nonetheless, HIV-1 interacts with DCs in a number of ways to promote virus spread (Geijtenbeek et al., 2000b; Sol-Foulon et al., 2002) (Figure 1A). For example, DCs express a number of attachment molecules which can mediate virus internalisation (Turville et al., 2002) as well as virus transfer to other cell types, such as $\mathrm{CD}^{+} \mathrm{T}$ lymphocytes (Lambert et al., 2008). For instance The c-type lectin dendritic cell-specific intercellular adhesion molecular 3-grabbing nonintegrin (DC-SIGN) is thought to play an important role in the entry of HIV-1 into cells (Feinberg et al., 2001). In DCs expressing both CD4 and DC-SIGN, the HIV-1 Env preferentially binds to DC-SIGN tetramers, which have been described as a "docking site" for HIV-1 (Cambi et al., 2004; Geijtenbeek et al., 2000b; Izquierdo-Useros et al., 2014). Moreover, the cytoplasmic region of DC-SIGN contains an internalization motif, and upon binding, the virus can be endocytosed into clathrin-coated pits (Cambi et al., 2009; Engering et al., 2002; Kwon et al., 2002). This process is thought to be dependent on clathrin, DNM2, and membrane cholesterol (Cambi et al., 2009; Ménager and Littman, 2016). HIV-1 may also 
be internalised by DCs through interactions with caveolin or processes involving bleb retraction and macropinocytosis, with the latter thought to be the primary method of virus entry (Ménager and Littman, 2016).

The fate of HIV-1 following internalization into DCs may depend on the mode of entry into the cell and has not been fully elucidated. Ratio fluorescence imaging has revealed that, in mature DCs, antigen-bound DC-SIGN localises to compartments with a neutral pH located close to the cell surface which may represent early endosomes (Cambi et al., 2004). In contrast, in immature DCs, internalised DC-SIGN localises to large compartments close to the nucleus with a low pH, characteristic of lysosomes (Engering et al., 2002). Within lysosomes, HIV-1 might be targeted for degradation and processed for presentation by the major histocompatibility complex (MHC) in T cells (Leonard et al., 2011). However, HIV-1 internalised by mature DCs is presumably protected from this pathway, observed by fluorescence confocal microscopy (Kwon et al., 2002); in these experiments DC cells were exposed to HIV-1 viruses at low temperature and then labelled with anti-HIV serum and fluorescently labelled transferrin, after shifting to $37{ }^{\circ} \mathrm{C}$, most of the HIV fluorescence was colocalized with the fluorescent transferrin in a sac-like nonlysosomal (Figure 2). Indeed, HIV1 has been observed to remain stable and infectious in mature DCs for many days following entry into the cell (Kwon et al., 2002).

Although the frequency of productive infection of DCs is low, it has been proposed that they may serve as virus reservoirs mediating infection of $\mathbf{C D 4}^{+} \mathbf{T}$ cells, and hence facilitate the spread of HIV-1 to lymph nodes (Wu and KewalRamani, 2006). HIV-1 can be transmitted from DCs to $\mathrm{CD}^{+}{ }^{+} \mathrm{T}$ cells in a number of ways, collectively referred to as trans-infection or trans-enhancement (Figure 1B) (Ménager and Littman, 2016). DC-SIGN-bound HIV-1 on the surface of DCs may be transported to lymph nodes by these DCs, where it can be brought into close proximity with CD4 and the CCR5/CXCR4 co-receptors on T cells (Hijazi et al., 2011; Wilen et al., 2012). HIV-1 can also be transferred between DCs and T cells by exocytosis of captured virus particles or via transmission of HIV-1 through the virological synapse, which forms upon direct contact between DCs and T cells (Ellwanger et al., 2016). Of note, this has been observed following extension of $\mathrm{CD}^{+}{ }^{+}$-rich filopodia from the $\mathrm{T}$ cell into HIV-1containing compartments located on DCs; the DC-T cell contact is thought to be mediated by 151 the binding of DC-SIGN to ICAM-3 on the CD4 ${ }^{+}$T cell (Felts et al., 2010; Geijtenbeek et al., 152 2000a). 
154 A recent paper published by Herold and colleagues (Herold et al., 2014) established that HIV-

1551 fuses in the plasma membrane of primary T cells. The authors concluded that endocytosed

156 viruses do not lead to productive HIV-1 fusion. This was shown using a dominant-negative 157 mutant that could block HIV-1 uptake by endocytosis but not HIV-1 fusion. The authors also 158 used an impermeable HIV-1 fusion inhibitor (T20) employed in primary CD4 T cells 159 preincubated at $22{ }^{\circ} \mathrm{C}$ (which allowed for HIV-1 endocytosis and not HIV-1 fusion). T20 turned 160 out to fully block HIV-1 fusion when raising the temperature at $37{ }^{\circ} \mathrm{C}$, indicating that 161 endocytosed virus were unable to fuse. In contrast, Miyauchi and co-workers (Miyauchi et al., 162 2009a) suggested that HIV-1 virus fuses within endosomes in TZM-bl and CEM cells as it can 163 engage CD4 and co-receptors at low temperatures but the rate of escape from T20 as compared 164 to temperature rise was delayed when performing population-based bulk HIV-1 fusion 165 experiments (Marin and Melikyan, 2015). The same group also showed that DNM2 inhibitor 166 dynasore (Kirchhausen et al., 2008) can block fusion (de la Vega et al., 2011; Miyauchi et al., 167 2009a; Miyauchi et al., 2009b). Moreover they visualized the process of fusion in endosomes 168 in TZM-bl reporter cells using double color real-time single particle tracking; however the 169 authors did not use primary CD4 T cells in their imaging experiments and therefore both studies 170 do not necessarily contradict each other. In a recent paper, we (Jones et al., 2017) and others 171 (Aggarwal et al., 2017) have shown that DNM2 is crucial for HIV-1 fusion in primary CD4 T 172 cells and it is not involved in endocytosis. Using advanced microscopy and functional-based 173 assays for HIV-1 fusion, we described the role of DNM2 during HIV-1 entry in TZM-bl cells 174 and primary CD4 T cells showing that DNM2 played a crucial role, establishing hemi-fusion 175 and in a later stage stabilizing the fusion pore, most likely at the plasma membrane. These data, 176 were further supported by Aggarwal and co-workers (Aggarwal et al., 2017). Combining 177 dynamin inhibition with functional HIV-1 fusion assays (Jones and Padilla-Parra, 2016); real178 time single HIV-1 tracking (Padilla-Parra et al., 2013); detection of HIV DNA products (van 179 Wilgenburg et al., 2014) the authors of this recent study identified that dynamin in CD4 T cells 180 directly regulates the fusion reaction at the plasma membrane. In conclusion, in recent years it 181 has been established by us and two other groups that HIV-1 most likely fuses in the plasma 182 membrane of primary CD4 T cells, and also, that the role of dynamin in these cells is strictly 183 related to the HIV-1 fusion reaction and not endocytosis. 


\section{Cell-to-cell transmission of HIV-1}

186 Contacts between infected cells and neighbouring uninfected target cells can enhance HIV-1

187 fusion and productive infection through the formation of the virological synapse (VS) (Groot 188 et al., 2008; Jolly et al., 2004; Jolly and Sattentau, 2007). During the establishment of the VS, 189 host and viral proteins are recruited to the site of intercellular contact, such as viral Gag and 190 Env from the donor cell, and CD4 in the target cell (Murooka et al., 2012). Initial Env-CD4 191 interactions can then lead to a second stage of VS stabilization in which other molecules interact at contact sites: e.g. Lymphocyte function-associated antigen-1 (LFA-1) and Intercellular Adhesion Molecule-1 (ICAM-1) (Li et al., 2015), which enhance adhesion between donor and target cell. Subsequently, Gag is recruited to the contact site of the infected cell and virion budding occurs into the interstitial space of the synapse (Hubner et al., 2009). How this process is regulated and the exact mechanism by which cell-cell fusion is avoided during the establishment of the VS is unknown but a subject of intense debate (Compton and Schwartz, 2017).

One report showed that HIV-1 could be taken up by DNM2-dependent endocytosis using both CXCR4- and CCR5-tropic viruses during cell-to-cell transmission between $\mathrm{CD}^{+}{ }^{+} \mathrm{T}$ cells (Sloan et al., 2013). The authors employed both RNA interference and small molecule inhibition in primary CD4 T cells to describe the role of DNM2 in productive cell to cell HIV1 transmission. In the same study, the authors observed, using a pharmacological and RNAi approach in primary CD4 + T cells, that certain transmission events were DNM2-dependent but not clathrin-dependent, suggesting that DNM2 might regulate HIV-1 cell-cell transmission independently of endocytosis (Sloan et al., 2013); this suggests that in HIV-1 cell to cell transmission, DNM2 might also be implicated in the fusion reaction, --as is the case in single virus entry in CD4 T cells (Jones et al., 2017), -- and not necessarily in endocytosis.

The role of endocytosis in cell-to-cell transmission is also a matter of debate. One study reported that HIV-1 virus transfer, fusion, and productive infection in target primary $\mathrm{CD}^{+} \mathrm{T}$ cells were not blocked by dynasore administration at sub-toxic concentrations (Kirchhausen et al., 2008), implying that endocytosis does not appear to constitute a major entry pathway in this setting (Permanyer et al., 2012). These results, however, contradict single virus tracking data in which HIV-1 was found to cross the VS and accumulate in endosomal compartments in target primary CD4 T cells (Dale et al., 2011). Furthermore, they proposed, based on data 
undergo maturation and posterior fusion in these compartments, meaning that immature HIV1 viruses could undergo maturation in endosomal compartments of the target cell (Dale et al., 2011). More data coming from tracking single double labelled HIV-1 virions with confocal microscopy also suggested that membrane fusion within endosomes might present delayed kinetics (several hours) to allow the proteolytic maturation of Gag to occur (Dale et al., 2011). This is important because endosomal delivery of virus following cell-to-cell transmission could serve to evade neutralization by circulating HIV-1-specific antibodies. To summarize, there is also a certain degree of controversy on the role of endocytosis during the establishment of the VS for HIV-1 cell to cell transmission (Permanyer et al., 2012) (Dale et al., 2011). In this context, DNM2 seems also to be crucial (Sloan et al., 2013), but its role could not be strictly related to endocytosis but perhaps in the HIV-1 fusion reaction; as is the case in single HIV-1 entry in CD4 T cells (Jones et al., 2017).

\section{Roles of DNM2 in HIV-1 fusion during infection}

DNM2 is known to contribute to cellular endocytosis by pinching the clathrin-coated pit upon forming an octameric, ring-like structure that is important for its GTPase activity (Ferguson and De Camilli, 2012). The finding that DNM2 contributes to HIV-1 entry was inferred to suggest that HIV-1 might enter cells via endocytosis (Miyauchi et al., 2009a). However, as explained above, recent reports have shown that DNM2 is directly involved in the virus-cell fusion process (Jones et al., 2017) (Aggarwal et al., 2017). The transition to full fusion or fission of two lipid bilayers depends on the competition between fusion and a calcium/ DNM2dependent fission mechanism (Artalejo et al., 2002; Chiang et al., 2014; Jaiswal et al., 2009; Zhao et al., 2016). This transition is very slow (seconds to minutes) and stresses the importance of the hemi-fusion state to regulate the transition towards full fusion (Zhao et al., 2016). A recent study demonstrated the importance of actin dynamics in this process, where actin must provide enough tension to merge fusing vesicles into the plasma membrane (Wen et al., 2016). This was shown by using confocal and super-resolution stimulated emission depletion (STED) microscopy, force measurements and gene knockouts. Indeed, filamentous actin, in conjunction with actin regulators N-WASP and Formin, seems to mediate the curvature between membranes favouring fusion by providing enough tension to transition toward full fusion of exosomes in chromaffin cells (Wen et al., 2016). In CD4+ T cells (Jones et al., 2017), hemi-fusion between HIV-1 and the plasma membrane of the host cell might evolve toward full fusion if two conditions are met: i) DNM2 forms a low oligomeric state, and ii) actin is selectively modulated to accommodate formation of the fusion pore (Yoder et al., 2008). 
Indeed, Yoder and co-workers (Yoder et al., 2008) showed by flow cytometry, western blot and kinase assays in resting CD4 $\mathrm{T}$ cells that cortical actin is a restriction factor overcome by HIV-1 by Gai-dependent signalling from the chemokine receptor (CXCR4) to activate the actin-depolymerizing factor cofilin (Yoder et al., 2008)

In DCs, however, the role of DNM2 is less clear and does not appear to be related to fusion. Moreover, DNM2 and tetraspanin (TRPM7) have been found to play an important role in promoting actin nucleation and stabilization allowing HIV-1 accumulation in dendrites, concomitantly avoiding HIV-1 macropinocytosis (Ménager and Littman, 2016). The importance of macropinocytosis during transenhancement was shown by employing several drug inhibitors selective for this particular endocytic process. The authors (Ménager and Littman, 2016) rescued HIV-1 trans-infection by DCs lacking DNM2; also reviewed in (Padilla-Parra and Dustin, 2016). In DCs the cortical actin cytoskeleton was shown to play a crucial role as opposed to endocytosis.

In addition to its role in early events of HIV-1 entry and fusion, DNM2 also regulates the fusogenicity of HIV-1 particles via its interaction with viral accessory protein Nef, in infected cells in Jurkat Tag lymphoid cells (Pizzatto et al., 2007). Furthermore, the incorporation of Serine Incoporator 3 and 5 (SERINC3 and SERINC5, known to be involved in serine incorporation into lipids and promote phosphatidylserine (PS) and sphingomyelin (SM) biosynthesis) into HIV-1 virions has been found to decrease virus infectivity in Jurkat cells and is antagonized by Nef (Rosa et al., 2015; Usami et al., 2015). Indeed, Usami and co-workers found that the infectivity of nef-deficient virions increased 100-fold in double-knockout primary CD4 T cells missing both SERINC3 and SERINC5 (Usami et al., 2015). In addition, in the presence of Nef, SERINC3 and SERINC5 can be sequestered from the cell surface to an endosomal compartment, thereby preventing incorporation into budding virions (Figure 3) (Usami et al., 2015). The mechanism by which SERINC proteins decrease fusogenicity is not understood, but recent evidence indicates that these proteins act immediately following fusion pore formation (Sood et al., 2017); this was shown using functional virus-cell and cell-cell fusion assays combined with single virus imaging using TZM-bl reporter cells, CV-1 cells stably expressing CD4 and CSCR4 and NSP/CD4/CXCR4/DSP-2 cells. A potential mechanistic clue comes from the observation that SERINC5 promotes the inactivation of Env glycoproteins by enhancing the exposure of gp41 domains (Sood et al., 2017). It now remains to be determined whether SERINC proteins or other inhibitors of lenvitiral fusogenicity (Compton et al. 2014) can have an impact on Env clustering and motility, processes which 
are crucial for HIV-1 engagement and productive fusion with an infected cell (Chojnacki et al., 2012) (Figure 4).

It is therefore clear that DNM2 has a multifaceted role during HIV-1 cell cycle: i) regulation of the fusion reaction in CD4 T cells (Aggarwal et al., 2017; Jones et al., 2017), ii) regulation of actin dynamics during trans-enhancement from DCs to CD4 T cells (Ménager and Littman, 2016) and iii) its role in downregulating SERINC3 and SERINC5 during egress and budding in primary CD4 $\mathrm{T}$ cells (Usami et al., 2015).

\section{Restriction by cell-intrinsic innate immunity influences the route of HIV-1 cellular entry}

Another potential explanation for the heterogeneity of sites available for HIV-1 fusion comes from studies of cell-intrinsic innate immune factors, such as the interferon-induced transmembrane (IFITM) proteins. IFITM proteins can inhibit numerous virus-cell and cellcell fusions at the entry step prior to fusion pore formation; mechanisms involved may include membrane deformation and altered lipid trafficking (Amini-Bavil-Olyaee et al., 2013; Desai et al., 2014; Li et al., 2013). A wide array of viruses are sensitive to IFITM-mediated antiviral activities, including HIV-1 (Compton et al., 2014; Compton et al., 2016; Lu et al., 2011) (Yu et al., 2015). In a recent study, the sensitivity of HIV-1 entry to IFITM-mediated restriction was reported to depend on co-receptor usage and the subcellular localisation of IFITM in the host cell (Foster et al., 2016). For example, X4 tropic HIV-1 strains were shown to exhibit sensitivity to IFITM2 and IFITM3, which are predominantly localized to endosomes and lysosomes, while R5 tropic strains were sensitive to IFITM1 at the plasma membrane of U87 neuroblastoma cells which do not express detectable IFITMs without IFN induction (Foster et al., 2016) (Figure 5). This differential susceptibility to different IFITM family members suggests that HIV-1 fusion might occur in endosomes or at the plasma membrane depending on which virus co-receptor is engaged at the cell surface. Moreover, Using transmitted/founder (TF) clones, which represent viruses that establish early infection following HIV-1 transmission, the authors showed that TF strains were resistant to IFITM-mediated restriction of virus entry (Foster et al., 2016). In addition, matched viral clones derived 6 months following initial infection exhibited a gain of sensitivity to endosomal IFITM2 and IFITM3. Further, When IFITM2 and IFITM3 were mutated to enforce stable plasma membrane localization, the ' 6 month' viruses remained resistant to their antiviral activities (Foster et al., 2016). Therefore, the progressive sensitization of primary virus isolates to IFITM2 and IFITM3 suggest that founder viruses enter cells at the plasma membrane while viruses isolated at later stages of 
infection might increasingly rely on endosomal entry (Foster et al., 2016) (Figure 5). Alternatively, since a pool of IFITM2 and IFITM3 is present at the plasma membrane prior to endosomal targeting via endocytosis, the differential sensitivities of HIV-1 strains to IFITM proteins might indicate that they could potentially fuse at different portions of the plasma membrane (Foster et al., 2016). The authors also show by infectivity assays that HIV-1 entry site choice, as inferred by sensitivity to IFITM, depends on the relative amounts of CD4 expressed in these cells (U87 neuroblastoma cells and primary CD4 T cells), thus linking receptor and co-receptor usage with viral entry route (Foster et al., 2016). Subsequent work further reinforced the link between IFITM and the port of entry used by HIV-1. A recent report demonstrated that X4 tropic HIV-1, but not R5 tropic, was hypersensitive to a splice variant of IFITM2 lacking the N-terminus (Wu et al., 2017). Jurkat E6-1 R5 cells expressing IFITM proteins were incubated with p24 antigen X4 tropic NL4-3 or R5-Tropic AD8 and virus titers measured as a function of time. R5 virus infection was unaffected (Wu et al., 2017). Since this IFITM variant was especially abundant in primary human cells (CD4 T cells and monocytes) susceptible to HIV-1 infection in vivo, the authors postulated that IFITM might limit X4 tropic infection, and thus, that it might contribute to the tendency of TF viruses to exhibit CCR5dependency during primary infection.

In brief, IFITM proteins might play an important role in the point of HIV-1 fusion. Understanding the different molecular mechanisms for the family of innate immunity of IFNinduced transmembrane (IFITM) proteins is very important to develop novel approaches to treat HIV-1 diseases. In particular the work of $\mathrm{Wu}$ and co-workers describing the specific restriction of Delat20 IFTM2 for CXCR4 (X4) tropism but not CCR5 (R5) may help to stop transmission of $\mathrm{X} 4$ viruses (acute phase).

\section{Concluding remarks}

In order to understand the restrictions imposed by different cell types on virus entry and fusion, it is crucial to explore the role of HIV-1 endocytosis and fusion globally in different tissues (CD4+ T cells, macrophages, and DCs) using different strategies. In fact, the heterogeneity of virus entry has to be addressed by studying each of the models mentioned above at molecular resolution, as the cell type and/or activation state of the target cell may impose different cytoskeletal and endocytic requirements. Describing these mechanisms might help us devise strategies to keep HIV-1 and other enveloped viruses at bay, and perhaps enable us to improve the efficiency of antiviral drugs and neutralizing antibodies. Here, we have stressed the 
problem of dissecting the port of HIV-1 entry in different cell lines and contexts and how cellular host proteins, such as DNM2, SERINC3, SERINC5 and IFITMs, might modulate this process together with the HIV-1 fusion reaction. We have discussed recent advances in the determination of the point of fusion of HIV-1 in primary $\mathrm{T}$ cells, the plasma membrane (Aggarwal et al., 2017; Herold et al., 2014; Jones et al., 2017) ) and primary macrophages (Li et al., 2017; Russell et al., 2017). We have reviewed the multi-dimensional role of DNM2: regulation during HIV-1 entry, fusion, and budding in different cellular contexts. These new insights constitute a challenging and promising field of research to potentially modulate host restriction factors and stop HIV-1 infection in the early stages. Clearly, many questions regarding HIV-1 cellular entry remain to be addressed (see Outstanding Questions and Box 3).

357 Furthermore, In order to design alternative and novel therapeutic interventions that focus on 358 the host cell rather than the virus itself, the current virus-centric approach must be reimagined. 359 The problem of heterogeneity in HIV-1 entry and fusion is therefore very complex and we need 360 to address it with an integrative approach using technologies from different disciplines: from advanced imaging approaches and tissue engineering to mathematical modelling.

362

\section{Acknowledgments}

364 This work has been supported by the Wellcome Trust to 203852/Z/16/2 to M.J 105278/Z/14/2 365 to R.N. The Wellcome Trust Centre for Human Genetics is funded by Wellcome Trust Core 366 Award 203852/Z/16/2 
369

370

371

372

373

374

375

376

377

378

379

380

381

382

383

384

385

386

387

388

389

390

391

392

393

394

395

396

397

398

399

400

401

402

403

404

405

406

407

408

409

410

411

412

\section{Actin}

Family of multifunctional proteins that form microfilaments. Actin participates in cell motility, cell division, cytokinesis, vesical and organelle movement, cell signalling and cell shape.

\section{Actin nucleation}

Actin filaments are generated by actin polymerization. The first step of this process is known as nucleation, and it's the formation of three actin monomers from which filaments may elongate.

\section{Actin remodelling}

Cellular process organized by actin monomers polymerization in response to signalling cascades that initiated by environment cues. The remodelling of actin filaments is mediated by actin-binding proteins and leads to change of the cell organization and shape.

\section{ART}

Anti-retroviral therapy. The gold standard in treatment of HIV-1, ART usually involves the combination of three antiviral drugs which act in different ways to limit spread of HIV-1. These may include fusion inhibitors, non-nucleoside reverse-transcriptase inhibitors (NNRTIs), nucleoside reverse-transcriptase inhibitors (NRTIs), protease inhibitors (PIs), entry inhibitors, or integrase strand transfer inhibitors (INSTIs). ART manages but does not cure HIV-1 infection.

\section{BET family inhibitors}

Protein inhibitors that reversibly bind proteins of the bromodomain and extra terminal motif family and prevent protein protein interactions between them and acetylated histones and transcription factors.

\section{Bispecific antibodies}

An artificial protein composed of two fragments of two different monoclonal antibodies or a monoclonal antibody and a neutralizing antibody.

\section{Bleb retraction}

Blebs are spherical cellular prostrations, not supported by an actin cytoskeleton, in mammalian cells observed during cytokinesis, apoptosis and cell migration. Retraction happens when cell's membrane lacks the stability afforded by cell-substrate contacts.

\section{CCR5}

7- transmembrane $\mathrm{G}$ protein coupled chemokine receptor for inflammatory chemokines. Upon binding it transduces a signal for increase of intracellular calcium ion level. It acts like a co-receptor (CD4 is the primary) for HIV R5 strains.

\section{CD4}

Membrane protein involved in the MHC- T cell interaction. It is the primary receptor for HIV-1.

\section{$\mathrm{CD4}^{+} \mathrm{T}$ cells}

Mature T cells with CD4 protein on their cell surface. They are the main targets of HIV-1.

\section{CEM cells}

T lymphoblastoid human cell line.

\section{Chromaffin cells}

Neuroendocrine cells part of the medulla of the adrenal glands in mammals. They are related to the sympathetic nervous system and they can migrate to sympathetic ganglia and to adrenal 
medulla. They secrete adrenaline, dopamine, encephalin and other hormones into the blood stream.

\section{Cofilin}

Protein which binds and disassembles actin filaments.

\section{Cortical actin network}

The richest area of actin filaments are found in a narrow zone underneath the plasma membrane (cortex). Most filaments there are organized into a network that excludes most organelles from the cortical cytoplasm.

\section{CRISPR/cas9 gene editing system}

Gene editing system based on the delivery of Cas9 endonuclease complexed with a synthetic guided RNA into a cell. Based on specific nucleotide repeats can alter the cell's genome at a desired location by allowing existing genes to be removed and new ones to be added.

\section{CXCR4}

Chemokine receptor with 7-transmembrane regions and part of the G protein coupled receptors. It acts as a co-receptor (CD4 is the primary) for HIV X4 strain.

\section{Dendrites}

Branched projections of a neuron that act to propagate the electrochemical stimulation received from other neural cells to the cell body of the neuron from which they project.

\section{Dendritic cells}

Professional antigen presenting cells located in numerous tissues (eg. skin, mucosal, lymphoid) throughout the body. They process antigens and then they present them on their cell surface to cytotoxic $\mathrm{T}$ cells while they express molecules as cytokines, chemokines and proteases to initiate immune response.

\section{Dendritic cell maturation}

Their ability to regulate immunity depends on their maturation. The process of maturation is based on phenotypical and functional changes and involves redistribution of MHC complex from intracellular compartments to their surface, down regulation of antigen internalization, cytoskeleton re-organization, secretion of cytokines, chemokines, proteases and surface expression of adhesion molecules and chemokine receptors.

\section{Dynamin-2}

Protein of the dynamin family, subfamily of the GTP-binding proteins. Dynamin-2 is found in all cell types. They are implicated in endocytosis, cell motility and in membrane alterations.

\section{Dynasore}

\section{Small GTPase inhibitor that targets dynamin-1 and dynamin-2.}

\section{Endocytosis}

The cell process of uptake of solid particles, small molecules or fluid droplets in small vesicles from the surrounding medium. It is divided in Clathrin mediated and non-clathrin mediated endocytosis.

\section{Env}

Viral envelope proteins encoded by the env gene. Env proteins are type I fusion machines thus bind to receptors at the cell membrane. This action triggers conformational changes allowing for binding of the fusion peptide. The env gene encodes for the precursor gp160 protein that is then cleaved to gp120 and gp41. 
$459 \quad$ Env clustering and motility

460 Trimeric Env proteins are distributed in the viral envelope. Studies have correlated the

461 maturation stage of Gag protein with the Env mobility and proposed that Env proteins can be

462 polarized towards CD4 receptors.

463 Filopodia

464 Slender bundled actin containing plasma membrane protrusions. They are important for cell

465 adhesions, migration and cell-to-cell contact.

466 Follicular CD8 $^{+} \mathbf{T}$ cells

467 HIV-1 specific cytolytic T cells usually found in the B cell follicles.

468 Founder Virus Clone

469 A single viral variant that initiates the HIV-1 infection.

470 Fusion Assays

471 Experimental procedures to test membrane fusion of enveloped viruses onto target cells, or

472 cells to other cells.

473 HIV-1

474 Human immunodeficiency virus type 1. An envelope lentivirus of the retroviridae family.

475 HIV-1 infection in humans leads to acquired immunodeficiency syndrome (AIDS).

476 HIV-1 R5

477 Viral strain that can infect cells expressing CD4 and CCR5 on their cell surface.

$478 \quad$ HIV-1 X4

479 Viral strain that can infect cells expressing CD4 and CXCR4 on their cell surface.

480 Histone Deacetylase inhibitors

481 Drugs with important role in epigenetic and non-epigenetic transcriptional regulation. In

482 HIV-1 reactivation they induce transcription at the HIV-1's long terminal repeat.

483 Histone methylotransferase inhibitors

484 Drugs for inhibiting known methylotransferases from methylating specific lysines of histones

485 and thus repressing HIV-1 transcription.

486 iPSCs

487 Induced pluripotent stem cells.

488 IFITMs

489 Interferone induced transmembrane proteins that inhibit virus entry and fusion in cells.

490 Internalization motif

491 Short linear motifs in the cytosolic tails of membrane proteins, that bind transiently to

492 proteins and protein complexes.

493 Interferones

494 Signalling proteins/cytokines released by cells of the immune system in response to presence

495 of pathogens.

496 Latent viral reservoirs

497 A group of long-lived cells that are infected with HIV but are not actively expressing it, as

498 patients undergo ART.

499 Latency reversing agents

500 Drugs used to induce HIV-1 expression in cells of patients under ART or in vitro.

501 Lentivirus

502 Genus of retroviruses that cause chronic and deadly diseases.

503

504 


\section{Lipid raft}

Different lipids present in plasma membrane have different biophysical propensities to associate with each other and form distinct and stable domains in membranes. Their existence it is still an area of debate.

\section{Macrophages}

White blood cells of the myeloid lineage that arise from the differentiation of monocytes. Macrophages engulf and destroy cells and materials that are recognised as foreign or damaged.

\section{Major Histocompatibility Complex}

Host proteins that are part of the adaptive immune system in all jawed vertebrates. They present antigens from intracellular proteins of infected or malignant cells to the cell's surface, where they can be surveyed by cytotoxic T lymphocytes.

\section{Macropinocytosis}

Is a form of endocytosis and it depends on actin cytoskeleton. It is the uptake of fluid droplets and it occurs in many cell types.

\section{Membrane Fission}

The process of splitting of a single lipid bilayer into two parts.

\section{Membrane Full Fusion}

The process where two distinct lipid bilayers merge to become one, also called closed fusion.

\section{Membrane Hemi-Fusion}

The intermediate state during membrane fusion when only the outer bilayer leaflets are mixed.

\section{Neutralizing antibodies}

Antibodies that are capable of blocking the virus by neutralization. This process can be mediated by blocking of the virion binding on cell receptors, block virus uptake from cells, prevent uncoating of viral genomes in endosomes or promote aggregation of virions.

\section{Nucleocapsid}

\section{The viral genome and the protein capsid that surrounds it.}

\section{N-WASP}

Neural Wiskott-Aldrich syndrome protein. It is a member of the WAS proteins which mediate signal transduction and interact with actin-binding proteins.

\section{Pore expansion}

During membrane fusion between virus and cell membranes a pore is created and that is the spot that the viral genome gains access to the host cell's cytoplasm. This process requires time and energy until the pore achieves the optimal width.

\section{Pre-integration complex (PIC)}

Nucleoprotein complex of viral genetic material associated with viral and host proteins capable of inserting the viral genome into the host genome.

\section{Ratio Fluorescence imaging}

Spectroscopic technique expressing the relationship of signals at two or more wavelengths.

\section{Recombination (DNA)}

\section{Exchange of DNA strands to produce new nucleotide arrangements.}

\section{Reporter cells}

Genetically modified cell line that stably express a reporter gene of interest expressed at the endogenous level for rapid monitoring

\section{Restriction factors}


551 Cellular proteins of the host that can serve as powerful blockades to HIV entry or replication.

552 Reverse Transcription

553 Synthesis of a DNA molecule from an RNA template.

554 Secretory pathway

555 It is a set of organelles of a cell like endoplasmic reticulum, Golgi apparatus and the vesicles

556 that travel between them and to the cell membrane or to the lysosomes. It is the pathway that

557 includes the synthesis and delivery of proteins to cell membrane, lysosomes and the secretory

558 organelles itself.

559 SERINC 3 and SERINC5

560 Serine incorporator 3 and 5. They enhance the incorporation of serine into phospatidylserine

561 and sphingolipids. They are also restriction factors of lentiviruses acting on an early step of

562 infection.

563 SIV

564 Simian immunodeficiency virus. SIV is a member of the retroviridae family that infects non-

565 human primates.

566 Tetraspasins

567 Protein transmembrane family involved in cell membrane compartmentalization, trafficking

568 regulation.

569 Trans Enhancement (HIV-1)

570 Infection of CD4 ${ }^{+}$T cells with HIV-1 from infected Dendritic Cells when co-cultured. The

571 viral particles have been internalized in a DC compartment and the transmission has been

572 proposed to occur in the vicinity of a DC-T-cell contact known as the infectious synapse.

573 Trans infection (HIV-1)

574 The infection of a host cell, a T cell for example by a different type of cell, like a Dendritic

575 Cell.

576 Viral DNA expression assays

577 Experimental procedures that identify if there is expression of the viral DNA in a host cell or 578 organism.

579 Viral Tropism

580 Virus specificity for particular cell types determined by the interaction of viral surface

581 proteins with host cell surface receptors.

582 Virological synapse

583 A tight adhesive junction between an HIV-infected cell and an uninfected target cell, across

584 which virus can be efficiently transferred without cell-cell fusion.

585

586

587

588

589

590

591

592 


\section{Box 1. HIV-1 Latent Reservoirs}

594

595

596

597

598

599

600

601

602

603

604

605

606

607

608

609

610

611

612

613

614

615

616

617

618

619

620

621

622

623

624

625

Individuals infected with HIV-1 are now able to live longer than ever before, and their health remains stable as HIV-1 infection is kept under control by anti-retroviral therapy (ART). However, HIV-1 infection remains a chronic illness due to the presence of latent viral reservoirs which persist within cells. The nature of these reservoirs has been best characterised in $\mathrm{CD}^{+} \mathrm{T}$ cells, however, the presence of viral reservoirs has also been observed in in various other cells and tissues, such as in the lymph nodes, gut-associated lymphoid tissue, the spleen, the liver, and the central nervous system (Henrich et al., 2017). HIV-1 is thought to accumulate in these reservoirs in the first few days of viremia, however, several studies have shown that replication of HIV-1 within lymphoid tissues is ongoing, even during ART treatment (LorenzoRedondo et al., 2016). A possible explanation for this observation has been attributed to unfavourable pharmacokinetics of commonly used anti-retroviral drugs, which might only reach the lymph nodes at low concentrations (Boritz and Douek, 2017). In an attempt to eradicate these latent HIV-1 reservoirs, the so-called "shock and kill" strategy has been developed (Box 2).

A number of methods are being considered to address the problems associated with reactivating latent reservoirs, such as using improved latency reversing agents (LRAs), neutralizing antibodies, bispecific antibodies, and by engineering $\mathrm{T}$ cells using the CRISPR/Cas9 gene editing system (Shan and Siliciano, 2013). Most LRAs target epigenetic factors; however, because viral reservoirs are present in a diverse range of cell types, the development of new small molecules or signalling molecules that act by altering the metabolism of cells may be required. Three to twelve months after the initiation of HIV-1 infection, broadly neutralising antibodies (bNAbs) are produced by the host to target parts of the virus. The identification of successful bNAbs produced by patients is currently the subject of extensive and promising research. The hope is that these bNAbs can be reproduced and combined to control viremia during ART interruption, engaging the host immune system to fight HIV-1 (Burton and Hangartner, 2016; Caskey et al., 2016). It was recently shown that a bispecific antibody acting as an bNAb recognising CD3 was able to increase the percentage of follicular $\mathbf{C D 8}^{+} \mathbf{T}$ cells, which killed with HIV-1 infected cells in vitro (Petrovas et al., 2017). Another putative therapeutic approach involves the use of the CRISPR/Cas9 gene editing system, which has been used to eliminate the HIV-1 genome from human T lymphoid cells (Kaminski et al., 2016). However, it has also been suggested that CRISPR/Cas9 derived mutations may accelerate viral escape (Wang et al., 2016a; Wang et al., 2016b). 
626 A main obstacle to the eradication of HIV-1 remains the presence of latent viral reservoirs.

627 These reservoirs are hidden inside a diverse range of cell types which have not been equally 628 represented in the literature. In order to address this, further research is required to identify the 629 relevant cell types, their locations, and the overall amount of latent viral DNA in HIV-1 630 infected patients.

\section{Box 2. The "Shock and Kill" Approach to HIV-1 Eradication}

632 The "shock and kill" approach involves activating HIV-1 transcription using agents that reverse 633 latency, such as deacetylase inhibitors, histone methyltransferase inhibitors, or BET 634 family inhibitors, thus transforming these cells into targets for the innate HIV-1 specific 635 immune system (Margolis and Archin, 2017; Siliciano, 2009). Interrupting ART in this way presents a number of risks. The viral genome is carried by a pool of different cell types and remains functional in some cells while being inactivated in others. Only a fraction of the cells carrying the functional viral genome will be reactivated by the "shock and kill" procedure, leaving behind the cells containing non-functional viral genomes, which present possible sites of viral rebound, following recombination. The "shock and kill" procedure may also pose a risk to healthy cells, leaving them liable for infection. Studies of SIV have shown that following the "shock and kill" approach, variants of the SIV genome that evade the immune system may reach the brain of non-human primates and might infect neighbouring cells (Gama et al., 2017). Furthermore, the immune cell response is not broad enough to tackle all reactivated cells.

Box 3. Clinician's corner.

New insights from restriction factors that inhibit virus-cell fusion may contribute to help in the potential development of future therapeutic interventions. Collectively, novel findings underline the need for potent, host-directed therapies that disrupt the earliest stages of the virus life cycle and preclude the emergence of resistant viral variants.

- A deep understanding of the HIV-1 fusion ports in different cell lines will impact the development of anti-HIV-1 agents (i.e. chemotherapeutic attack). With novel insights on the molecular mechanisms of HIV-1 entry three different type of inhibitors can be improved: i) attachment inhibitors, ii) co-receptor binding inhibitors and iii) fusion inhibitors. 
- A number of strategies have been tried to block interactions between Env gp120 and CD4. For example, soluble CD4 that induces a non-fusogenic conformation of gp120, small molecule inhibitors targeting the CD4-gp120 binding site or CD4 antibodies. All these approaches will benefit from knowing the exact point of fusion in different cellular contexts.

- It has been reported that in $1 \%$ of the Caucasian population naturally occurs a homozygous deletion in the CCR5 allele not associated with immunological dysfunction. This leaded to the development of Maraviroc, which was approved by the US Food and Drud Administration (FDA) and the European Medicine Agency to treat patients with multi-resistant HIV-1 strains. This is, so far, the only example of a drug targeting a HIV-1 cellular factor in the clinic, that in this case is related to entry. A deep understanding of the molecular mechanisms that lead to HIV-1 fusion and the exact point of entry could help to develop other drugs targeting host restriction factors.

670

671

672

673

674

675 
Aggarwal, A., Hitchen, T.L., Ootes, L., McAllery, S., Wong, A., Nguyen, K., McCluskey, A., Robinson, P.J., and Turville, S.G. (2017). HIV infection is influenced by dynamin at 3 independent points in the viral life cycle. Traffic 18, 392-410. Amini-Bavil-Olyaee, S., Choi, Y.J., Lee, J.H., Shi, M., Huang, I.-C., Farzan, M., and Jung, J.U. (2013). The antiviral effector IFITM3 disrupts intracellular cholesterol homeostasis to block viral entry. Cell host \& microbe 13, 452-464.

685 endocytosis from dynamin-1-dependent rapid endocytosis to clathrin- and dynamin-2-mediated slow endocytosis in chromaffin cells. Proc Natl Acad Sci U S A 99, 6358-6363.

Boritz, E.A., and Douek, D.C. (2017). Perspectives on Human Immunodeficiency Virus (HIV) Cure: HIV Persistence in Tissue. The Journal of Infectious Diseases 215, S128-S133.

Burton, D.R., and Hangartner, L. (2016). Broadly neutralizing antibodies to HIV and their role in vaccine design. Annual review of immunology 34, 635-659.

Cambi, A., Beeren, I., Joosten, B., Fransen, J.A., and Figdorl, C.G. (2009). The C-type lectin DC-SIGN internalizes soluble antigens and HIV-1 virions via a clathrin-dependent mechanism. Eur J Immunol 39, 1923-1928.

Cambi, A., de Lange, F., van Maarseveen, N.M., Nijhuis, M., Joosten, B., van Dijk, E., de Bakker, B.I., Fransen, J.A.M., Bovee-Geurts, P.H.M., van Leeuwen, F.N., et al. (2004). Microdomains of the C-type lectin DC-SIGN are portals for virus entry into dendritic cells. J Cell Biol 164, 145-155.

Carter, G.C., Bernstone, L., Baskaran, D., and James, W. (2011). HIV-1 infects macrophages by exploiting an endocytic route dependent on dynamin, Rac1 and Pak1. Virology 409, 234-250.

Caskey, M., Klein, F., and Nussenzweig, M.C. (2016). Broadly Neutralizing Antibodies for HIV-1 Prevention or Immunotherapy. New England Journal of Medicine 375, 2019-2021.

Chiang, H.C., Shin, W., Zhao, W.D., Hamid, E., Sheng, J.S., Baydyuk, M., Wen, P.J., Jin, A., Momboisse, F., and Wu, L.G. (2014). Post-fusion structural changes and their roles in exocytosis and endocytosis of dense-core vesicles. Nat Commun 5, 15.

Chojnacki, J., Staudt, T., Glass, B., Bingen, P., Engelhardt, J., Anders, M., Schneider, J., Muller, B., Hell, S.W., and Krausslich, H.G. (2012). Maturation-Dependent HIV-1 Surface Protein Redistribution Revealed by Fluorescence Nanoscopy. Science 338, 524-528.

Compton, A.A., Bruel, T., Porrot, F., Mallet, A., Sachse, M., Euvrard, M., Liang, C., Casartelli, N., and Schwartz, O. (2014). IFITM Proteins Incorporated into HIV-1 Virions Impair Viral Fusion and Spread. Cell Host Microbe 16, 736-747.

Compton, A.A., Roy, N., Porrot, F., Billet, A., Casartelli, N., Yount, J.S., Liang, C., and Schwartz, O. (2016). Natural mutations in IFITM3 modulate post-translational regulation and toggle antiviral specificity. EMBO Rep 17, 1657-1671.

Compton, A.A., and Schwartz, O. (2017). They Might Be Giants: Does Syncytium Formation Sink or Spread HIV Infection? PLoS Pathog 13, 7.

Dale, B.M., McNerney, G.P., Thompson, D.L., Hubner, W., de los Reyes, K., Chuang, F.Y.S., Huser, T., and Chen, B.K. (2011). Cell-to-Cell Transfer of HIV-1 via Virological Synapses Leads to Endosomal Virion Maturation that Activates Viral Membrane Fusion. Cell Host Microbe 10, 551-562.

de la Vega, M., Marin, M., Kondo, N., Miyauchi, K., Kim, Y., Epand, R.F., Epand, R.M., and Melikyan, G.B. (2011). Inhibition of HIV-1 endocytosis allows lipid mixing at the plasma membrane, but not complete fusion. Retrovirology 8.

Desai, T.M., Marin, M., Chin, C.R., Savidis, G., Brass, A.L., and Melikyan, G.B. (2014). IFITM3 Restricts Influenza A Virus Entry by Blocking the Formation of Fusion Pores following Virus-Endosome Hemifusion. PLoS Pathog 10, 16. 
Ellwanger, J.H., Crovella, S., dos Reis, E.C., Pontillo, A., and Chies, J.A.B. (2016). Exosomes are possibly used as a tool of immune regulation during the dendritic cell-based immune therapy against HIV-I. Med Hypotheses 95, 67-70.

Engering, A., Geijtenbeek, T.B.H., van Vliet, S.J., Wijers, M., van Liempt, E., Demaurex, N., Lanzavecchia, A., Fransen, J., Figdor, C.G., Piguet, V., et al. (2002). The dendritic cell-specific adhesion receptor DC-SIGN internalizes antigen for presentation to T cells. J Immunol 168, 2118-2126.

Feinberg, H., Mitchell, D.A., Drickamer, K., and Weis, W.I. (2001). Structural basis for selective recognition of oligosaccharides by DC-SIGN and DC-SIGNR. Science 294, 2163-2166.

Felts, R.L., Narayan, K., Estes, J.D., Shi, D., Trubey, C.M., Fu, J., Hartnell, L.M., Ruthel, G.T., Schneider, D.K., and Nagashima, K. (2010). 3D visualization of HIV transfer at the virological synapse between dendritic cells and T cells. Proceedings of the National Academy of Sciences 107, 13336-13341.

Ferguson, S.M., and De Camilli, P. (2012). Dynamin, a membrane-remodelling GTPase. Nature Reviews Molecular Cell Biology 13, 75-88.

Foster, T.L., Wilson, H., Iyer, S.S., Coss, K., Doores, K., Smith, S., Kellam, P., Finzi, A., Borrow, P., Hahn, B.H., et al. (2016). Resistance of Transmitted Founder HIV-1 to IFITM-Mediated Restriction. Cell Host Microbe 20, 429-442.

Gallo, R.C. (2016). Shock and kill with caution. Science 354, 177-178.

Gama, L., Abreu, C.M., Shirk, E.N., Price, S.L., Li, M., Laird, G.M., Pate, K.A.M., Wietgrefe, S.W., O'connor, S.L., and Pianowski, L. (2017). Reactivation of simian immunodeficiency virus reservoirs in the brain of virally suppressed macaques. AIDS (London, England) 31, 5.

Gandhi, R.T., Zheng, L., Bosch, R.J., Chan, E.S., Margolis, D.M., Read, S., Kallungal, B., Palmer, S., Medvik, K., Lederman, M.M., et al. (2010). The Effect of Raltegravir Intensification on Low-level Residual Viremia in HIV-Infected Patients on Antiretroviral Therapy: A Randomized Controlled Trial. PLos Med 7, 11.

Geijtenbeek, T.B., Torensma, R., van Vliet, S.J., van Duijnhoven, G.C., Adema, G.J., van Kooyk, Y., and Figdor, C.G. (2000a). Identification of DC-SIGN, a novel dendritic cell-specific ICAM-3 receptor that supports primary immune responses. Cell 100, 575-585.

Geijtenbeek, T.B.H., Kwon, D.S., Torensma, R., van Vliet, S.J., van Duijnhoven, G.C.F., Middel, J., Cornelissen, I., Nottet, H., KewalRamani, V.N., Littman, D.R., et al. (2000b). DC-SIGN, a dendritic cellspecific HIV-1-binding protein that enhances trans-infection of T cells. Cell 100, 587-597.

Groot, F., Welsch, S., and Sattentau, Q.J. (2008). Efficient HIV-1 transmission from macrophages to T cells across transient virological synapses. Blood 111, 4660-4663.

Henrich, T.J., Deeks, S.G., and Pillai, S.K. (2017). Measuring the size of the latent human immunodeficiency virus reservoir: the present and future of evaluating eradication strategies. The Journal of Infectious Diseases 215, S134-S141.

Herold, N., Anders-Osswein, M., Glass, B., Eckhardt, M., Muller, B., and Krausslich, H.G. (2014). HIV-1 Entry in SupT1-R5, CEM-ss, and Primary CD4(+) T Cells Occurs at the Plasma Membrane and Does Not Require Endocytosis. J Virol 88, 13956-13970.

Hijazi, K., Wang, Y., Scala, C., Jeffs, S., Longstaff, C., Stieh, D., Haggarty, B., Vanham, G., Schols, D., and Balzarini, J. (2011). DC-SIGN increases the affinity of HIV-1 envelope glycoprotein interaction with CD4. PLoS One 6, e28307.

Honeycutt, J.B., Thayer, W.O., Baker, C.E., Ribeiro, R.M., Lada, S.M., Cao, Y.F., Cleary, R.A., Hudgens, M.G., Richman, D.D., and Garcia, J.V. (2017). HIV persistence in tissue macrophages of humanized myeloid-only mice during antiretroviral therapy. Nat Med 23, 638-+.

Hubner, W., McNerney, G.P., Chen, P., Dale, B.M., Gordon, R.E., Chuang, F.Y.S., Li, X.D., Asmuth, D.M., Huser, T., and Chen, B.K. (2009). Quantitative 3D Video Microscopy of HIV Transfer Across T Cell Virological Synapses. Science 323, 1743-1747.

Izquierdo-Useros, N., Lorizate, M., McLaren, P.J., Telenti, A., Krausslich, H.G., and Martinez-Picado, J. (2014). HIV-1 capture and transmission by dendritic cells: the role of viral glycolipids and the cellular receptor Siglec-1. PLoS Pathog 10, e1004146. 
Jaiswal, J.K., Rivera, V.M., and Simon, S.M. (2009). Exocytosis of Post-Golgi Vesicles Is Regulated by Components of the Endocytic Machinery. Cell 137, 1308-1319.

Jolly, C., Kashefi, K., Hollinshead, M., and Sattentau, Q.J. (2004). HIV-1 cell to cell transfer across an env-induced, actin-dependent synapse. Journal of Experimental Medicine 199, 283-293.

Jolly, C., and Sattentau, Q.J. (2007). Human immunodeficiency virus type 1 assembly, budding, and cell-cell spread in T cells take place in tetraspanin-enriched plasma membrane domains. J Virol 81, 7873-7884.

Jones, D.M., Alvarez, L.A., Nolan, R., Ferriz, M., Urruela, R.S., Massana-Munoz, X., Novak-Kotzer, H., Dustin, M.L., and Padilla-Parra, S. (2017). Dynamin-2 Stabilizes the HIV-1 Fusion Pore with a Low Oligomeric State. Cell Reports 18, 443-453.

Jones, D.M., and Padilla-Parra, S. (2016). The $\beta$-lactamase assay: Harnessing a FRET biosensor to analyse viral fusion mechanisms. Sensors (Switzerland) 16.

Kaminski, R., Chen, Y., Fischer, T., Tedaldi, E., Napoli, A., Zhang, Y., Karn, J., Hu, W., and Khalili, K. (2016). Elimination of HIV-1 genomes from human T-lymphoid cells by CRISPR/Cas9 gene editing. Scientific reports 6 .

Kirchhausen, T., Macia, E., and Pelish, H.E. (2008). Use of dynasore, the small molecule inhibitor of dynamin, in the regulation of endocytosis. In Small Gtpases in Disease, Part A, W.E. Balch, C.J. Der, and A. Hall, eds. (San Diego: Elsevier Academic Press Inc), pp. 77-93.

Kwon, D.S., Gregorio, G., Bitton, N., Hendrickson, W.A., and Littman, D.R. (2002). DC-SIGN-mediated internalization of HIV is required for trans-enhancement of T cell infection. Immunity 16, 135-144. Laguette, N., Rahm, N., Sobhian, B., Chable-Bessia, C., Munch, J., Snoeck, J., Sauter, D., Switzer, W.M., Heneine, W., Kirchhoff, F., et al. (2012). Evolutionary and Functional Analyses of the Interaction between the Myeloid Restriction Factor SAMHD1 and the Lentiviral Vpx Protein. Cell Host Microbe 11, 205-217.

Lambert, A.A., Gilbert, C., Richard, M., Beaulieu, A.D., and Tremblay, M.J. (2008). The C-type lectin surface receptor DICIR acts as a new attachment factor for HIV-1 in dendritic cells and contributes to trans- and cis-infection pathways. Blood 112, 1299-1307.

Lee, J.H., Ozorowski, G., and Ward, A.B. (2016). Cryo-EM structure of a native, fully glycosylated, cleaved HIV-1 envelope trimer. Science 351, 1043-1048.

Leonard, J.A., Filzen, T., Carter, C.C., Schaefer, M., and Collins, K.L. (2011). HIV-1 Nef disrupts intracellular trafficking of major histocompatibility complex class I, CD4, CD8, and CD28 by distinct pathways that share common elements. J Virol 85, 6867-6881.

Li, G.H., Anderson, C., Jaeger, L., Do, T., Major, E.O., and Nath, A. (2015). Cell-to-cell contact facilitates HIV transmission from lymphocytes to astrocytes via CXCR4. Aids 29, 755-766.

Li, K., Markosyan, R.M., Zheng, Y.M., Golfetto, O., Bungart, B., Li, M.H., Ding, S.L., He, Y.X., Liang, C., Lee, J.C., et al. (2013). IFITM Proteins Restrict Viral Membrane Hemifusion. PLoS Pathog 9, 18.

Li, Q., Li, W., Yin, W., Guo, J., Zhang, Z.P., Zeng, D.J., Zhang, X.W., Wu, Y.T., Zhang, X.E., and Cui, Z.Q. (2017). Single-Particle Tracking of Human Immunodeficiency Virus Type 1 Productive Entry into Human Primary Macrophages. ACS Nano 11, 3890-3903.

Lorenzo-Redondo, R., Fryer, H.R., Bedford, T., Kim, E.Y., Archer, J., Pond, S.L.K., Chung, Y.S., Penugonda, S., Chipman, J., Fletcher, C.V., et al. (2016). Persistent HIV-1 replication maintains the tissue reservoir during therapy. Nature 530, 51-56.

Lu, J., Pan, Q.H., Rong, L.W., Liu, S.L., and Liang, C. (2011). The IFITM Proteins Inhibit HIV-1 Infection. J Virol 85, 2126-2137.

Marechal, V., Prevost, M.C., Petit, C., Perret, E., Heard, J.M., and Schwartz, O. (2001). Human immunodeficiency virus type 1 entry into macrophages mediated by macropinocytosis. J Virol 75, 11166-11177.

Margolis, D.M., and Archin, N.M. (2017). Proviral Latency, Persistent Human Immunodeficiency Virus Infection, and the Development of Latency Reversing Agents. The Journal of Infectious Diseases 215, S111-S118. 
Marin, M., and Melikyan, G.B. (2015). Can HIV-1 Entry Sites Be Deduced by Comparing Bulk Endocytosis to Functional Readouts for Viral Fusion? J Virol 89, 2985-2985. Medlock, J., Pandey, A., Parpia, A.S., Tang, A., Skrip, L.A., and Galvani, A.P. (2017). Effectiveness of UNAIDS targets and HIV vaccination across 127 countries. Proc Natl Acad Sci U S A 114, 4017-4022. Mellman, I. (1996). Endocytosis and molecular sorting. Annu Rev Cell Dev Biol 12, 575-625. Ménager, M.M., and Littman, D.R. (2016). Actin Dynamics Regulates Dendritic Cell-Mediated Transfer of HIV-1 to T Cells. Cell 164, 695-709.

Miyauchi, K., Kim, Y., Latinovic, O., Morozov, V., and Melikyan, G.B. (2009a). HIV Enters Cells via Endocytosis and Dynamin-Dependent Fusion with Endosomes. Cell 137, 433-444.

Miyauchi, K., Kozlov, M.M., and Melikyan, G.B. (2009b). Early Steps of HIV-1 Fusion Define the Sensitivity to Inhibitory Peptides That Block 6-Helix Bundle Formation. PLoS Pathog 5.

Murooka, T.T., Deruaz, M., Marangoni, F., Vrbanac, V.D., Seung, E., von Andrian, U.H., Tager, A.M., Luster, A.D., and Mempel, T.R. (2012). HIV-infected T cells are migratory vehicles for viral dissemination. Nature 490, 283-+.

Padilla-Parra, S., and Dustin, M.L. (2016). Actin Dynamics and HIV-1 Entry. Trends in Molecular Medicine 22, 354-356.

Padilla-Parra, S., Marin, M., Gahlaut, N., Suter, R., Kondo, N., and Melikyan, G.B. (2013). Fusion of Mature HIV-1 Particles Leads to Complete Release of a Gag-GFP-Based Content Marker and Raises the Intraviral pH. PLoS One 8.

Parrish, N.F., Wilen, C.B., Banks, L.B., Iyer, S.S., Pfaff, J.M., Salazar-Gonzalez, J.F., Salazar, M.G., Decker, J.M., Parrish, E.H., Berg, A., et al. (2012). Transmitted/Founder and Chronic Subtype C HIV-1 Use CD4 and CCR5 Receptors with Equal Efficiency and Are Not Inhibited by Blocking the Integrin alpha 4 beta 7. PLoS Pathog $8,16$.

Permanyer, M., Ballana, E., Badia, R., Pauls, E., Clotet, B., and Este, J.A. (2012). Trans-infection but Not Infection from within Endosomal Compartments after Cell-to-cell HIV-1 Transfer to CD4(+) T Cells. J Biol Chem 287, 32017-32026.

Petrovas, C., Ferrando-Martinez, S., Gerner, M.Y., Casazza, J.P., Pegu, A., Deleage, C., Cooper, A., Hataye, J., Andrews, S., and Ambrozak, D. (2017). Follicular CD8 T cells accumulate in HIV infection and can kill infected cells in vitro via bispecific antibodies. Science translational medicine 9, eaag2285.

Piguet, V., and Steinman, R.M. (2007). The interaction of HIV with dendritic cells: outcomes and pathways. Trends Immunol 28, 503-510.

Rosa, A., Chande, A., Ziglio, S., De Sanctis, V., Bertorelli, R., Goh, S.L., McCauley, S.M., Nowosielska, A., Antonarakis, S.E., and Luban, J. (2015). HIV-1 Nef promotes infection by excluding SERINC5 from virion incorporation. Nature 526, 212-217.

Russell, R.A., Chojnacki, J., Jones, D.M., Johnson, E., Do, T., Eggeling, C., Padilla-Parra, S., and Sattentau, Q.J. (2017). Astrocytes Resist HIV-1 Fusion but Engulf Infected Macrophage Material. Cell Reports 18, 1473-1483.

Shan, L., and Siliciano, R.F. (2013). From reactivation of latent HIV-1 to elimination of the latent reservoir: the presence of multiple barriers to viral eradication. BioEssays : news and reviews in molecular, cellular and developmental biology 35, 544-552.

Siliciano, R. (2009). What do we need to do to cure HIV infection. Topics in HIV medicine: a publication of the International AIDS Society, USA 18, 104-108.

Simmons, G., Clapham, P.R., Picard, L., Offord, R.E., Rosenkilde, M.M., Schwartz, T.W., Buser, R., Wells, T.N.C., and Proudfoot, A.E.I. (1997). Potent inhibition of HIV-1 infectivity in macrophages and lymphocytes by a novel CCR5 antagonist. Science 276, 276-279.

Sloan, R.D., Kuhl, B.D., Mesplede, T., Munch, J., Donahue, D.A., and Wainberg, M.A. (2013). Productive Entry of HIV-1 during Cell-to-Cell Transmission via Dynamin-Dependent Endocytosis. J Virol 87, 81108123.

Sol-Foulon, N., Moris, A., Nobile, C., Boccaccio, C., Engering, A., Abastado, J.P., Heard, J.M., van Kooyk, Y., and Schwartz, O. (2002). HIV-1 nef-induced Upregulation of DC-SIGN in dendritic cells promotes lymphocyte clustering and viral spread. Immunity 16, 145-155. 
Sood, C., Marin, M., Chande, A., Pizzato, M., and Melikyan, G.B. (2017). SERINC5 protein inhibits HIV1 fusion pore formation by promoting functional inactivation of envelope glycoproteins. J Biol Chem 292, 6014-6026.

Turville, S.G., Cameron, P.U., Handley, A., Lin, G., Pohlmann, S., Doms, R.W., and Cunningham, A.L. (2002). Diversity of receptors binding HIV on dendritic cell subsets. Nat Immunol 3, 975-983. Usami, Y., Wu, Y.F., and Gottlinger, H.G. (2015). SERINC3 and SERINC5 restrict HIV-1 infectivity and are counteracted by Nef. Nature 526, 218-+. van Wilgenburg, B., Moore, M.D., James, W.S., and Cowley, S.A. (2014). The Productive Entry Pathway of HIV-1 in Macrophages Is Dependent on Endocytosis through Lipid Rafts Containing CD4. PLoS One 9.

Wang, G., Zhao, N., Berkhout, B., and Das, A.T. (2016a). CRISPR-Cas9 can inhibit HIV-1 replication but NHEJ repair facilitates virus escape. Molecular Therapy.

Wang, Z., Pan, Q., Gendron, P., Zhu, W., Guo, F., Cen, S., Wainberg, M.A., and Liang, C. (2016b). CRISPR/Cas9-derived mutations both inhibit HIV-1 replication and accelerate viral escape. Cell reports 15, 481-489.

Wen, P.J., Grenklo, S., Arpino, G., Tan, X.Y., Liao, H.S., Heureaux, J., Peng, S.Y., Chiang, H.C., Hamid, E., Zhao, W.D., et al. (2016). Actin dynamics provides membrane tension to merge fusing vesicles into the plasma membrane. Nat Commun 7, 14.

Wilen, C.B., Tilton, J.C., and Doms, R.W. (2012). HIV: cell binding and entry. Cold Spring Harbor perspectives in medicine 2, a006866.

Wu, B., Chien, E.Y., Mol, C.D., Fenalti, G., Liu, W., Katritch, V., Abagyan, R., Brooun, A., Wells, P., Bi, F.C., et al. (2010). Structures of the CXCR4 chemokine GPCR with small-molecule and cyclic peptide antagonists. Science 330, 1066-1071.

Wu, L., and KewalRamani, V.N. (2006). Dendritic-cell interactions with HIV: infection and viral dissemination. Nature Reviews Immunology 6, 859-868.

Wu, W.L., Grotefend, C.R., Tsai, M.T., Wang, Y.L., Radic, V., Eoh, H., and Huang, I.C. (2017). Delta20 IFITM2 differentially restricts $X 4$ and R5 HIV-1. Proc Natl Acad Sci U S A.

Yoder, A., Yu, D.Y., Dong, L., Iyer, S.R., Xu, X.H., Kelly, J., Liu, J., Wang, W.F., Vorster, P.J., Agulto, L., et al. (2008). HIV envelope-CXCR4 signaling activates cofilin to overcome cortical actin restriction in resting CD4 T cells. Cell 134, 782-792.

Yu, J.Y., Li, M.H., Wilkins, J., Ding, S.L., Swartz, T.H., Esposito, A.M., Zheng, Y.M., Freed, E.O., Liang, C., Chen, B.K., et al. (2015). IFITM Proteins Restrict HIV-1 Infection by Antagonizing the Envelope Glycoprotein. Cell Reports 13, 145-156.

Zaitseva, E., Zaitsev, E., Melikov, K., Arakelyan, A., Marin, M., Villasmil, R., Margolis, L.B., Melikyan, G.B., and Chernomordik, L.V. (2017). Fusion Stage of HIV-1 Entry Depends on Virus-Induced Cell Surface Exposure of Phosphatidylserine. Cell Host Microbe 22, 99-110.e117.

Zhao, W.D., Hamid, E., Shin, W., Wen, P.J., Krystofiak, E.S., Villarreal, S.A., Chiang, H.C., Kachar, B., and Wu, L.G. (2016). Hemi-fused structure mediates and controls fusion and fission in live cells. Nature $534,548-+$. 
Figure 1. HIV-1 cellular entry and dissemination in dendritic cells. A. HIV-1 can enter dendritic cells (DCs) in different ways, the most prolific ones being endocytosis and macropinocytosis. Upon binding, the HIV-1-DC-SIGN complex may be internalised into clathrin coated pits, pinched off through the action of dynamin. In macropinocytosis, sheetlike projections from the membrane engulf the virus and other materials present in the extracellular space, encapsulating them in a surface accessible compartment. Once internalised, virus-containing vesicles can be targeted for lysosomal degradation and processing for antigen presentation, or, may become early endosomes, protecting the virus. $\mathbf{B}$. HIV-1 can be transferred from DCs to its main target, $\mathrm{CD}^{+}{ }^{+} \mathrm{T}$ cells. Internalized viruses can be released extracellularly through exocytosis, infecting neighbouring cells. CD4+ T cells can also be infected by HIV-1 bound to DC-SIGN on DCs. CD4-rich filopodia projecting from the T cell can penetrate virus-containing compartments close to the surface of DCs, binding HIV1 virions which then "surf" along filopodia to the T cell body and infect it. HIV-1 can also be transferred across the virological synapse between the DC and the CD4+ T cell, protected by sheet-like projections from the DC, (trans-infection). C. In the virological synapse, cell surface receptors and viral glycoproteins are recruited to the contact site between the DC and the T cell, stabilised by interactions between LFA-1-ICAM-1, and DC-SIGN-ICAM-3.

Figure 2. Formation of the HIV-1 fusion pore. The formation of the HIV-1 fusion pore involves a number of well-defined intermediate conformational states. HIV-1 Env binds to the CD4 receptor and then engages with the CCR5 co-receptor, forming the "early prebundle". This state progresses to that of the "binding prebundle", which gives rise to the "stalk-pore" model of membrane fusion. First, the outer leaflets of the lipid bilayer connect, forming a stalk.

941 This leads to the "hemifusion" state, where the outer leaflets have fused but the inner halves of 942 the two lipid bilayers remain uncorrupted, but in close contact, in a structure known as the "fusogenic prebundle". Finally, the inner membranes fuse, creating the "fusion pore", which forms due to the arrangement of proteins into the "6-helix bundle". Dynamin is thought to play a role at various stages of fusion pore formation, such as facilitating the binding of co-receptors, inducing hemifusion, and stabilising the fusoin pore (2). Dynamin inhibitors, such as dynasore and TAK779, can inhibit the binding of the CCR5 co-receptor. Dynasore also interferes with 
the stabilisation of the fusion pore by dynamin. The formation of the fusion pore can be inhibited by synthetic peptides T20 (HIV-1 fusion inhibitor) and temperature block (TB).

Figure 3. HIV-1 Cellular Egress. A. (1) Nef interacts with dynamin-2 (DNM2).

Activation of DMN2 by Nef facilitates clathrin mediated endocytosis of the CD4 and CCR5 receptors into clathrin coated pits. (3) Activation of DNM2 by Nef also results in (4) sequestration of SERINC3 and SERINC5 from the plasma membrane. DNM2 is involved in the scission of these endocytic vesicles from the membrane. The decrease in SERINC3/SERINC5 present at the plasma membrane results in the egress of fusion competent SERINC3/5 deficient virions in CD4 T cells. B. Inhibition (with mutants or RNA interference) of the (1) Nef-dynamin interaction results in the (5) release of fusion non -competent viruses with SERINC3/5 embedded in the viral envelope. CME: Clathrin mediated endocytosis

Figure 4. Structure and formation of HIV-1 particles. In mature HIV-1 particles the Env glycoproteins are able to move around in the viral membrane and can arrange themselves in trimers. In immature HIV-1 particles, Env motility is reduced due to the immatureGag lattice around RNA. This inhibits the ability of immature HIV-1 to undergo fusion with a cell membrane.

Figure 5. Role of IFITMs in HIV-1 restriction of entry and fusion. HIV-1 entry (left hand side of the cartoon, dashed line) occurs in CD4 T cells via R5 tropic viruses (R5 Env, blue) via interaction with CD4 and CCR5 and fusion occurs in the plasma membrane. HIV-1 X4 tropic viruses (X4 Env, green) occurs via interaction with CD4 and CXCR4 and internalization via endocytosis occurs. The virus might fuse within endosomes in the absence of IFITM2/3. IFITM2/3 proteins (red) present in endosomes might restrict HIV-1 fusion. The process of HIV-1 egress (right hand side of the cartoon, dashed line) is also subject to IFTM2/3 restriction. In this case, only the X4 tropic viruses with IFITM2/3 incorporated will be fusion defective. On the contrary, the R5 tropic HIV-1 virus with IFITM2/3 incorporated in its envelope will be fusion competent. In the particular case of $293 \mathrm{~T}$ cells IFITM2/3 will also restrict X4 tropic HIV-1 viruses and therefore these viruses will be fusion defective. 